BERNHARD METZ

\title{
Zur Edierbarkeit großer Formen. Über (un)mögliche Editionsprojekte
}

I. Große Form und Lebenskürze. Dass es (literarische) Texte und große Formen gibt, die allein aufgrund ihres schieren Umfangs bzw. quantitativer Aspekte ernsthafte Herausforderungen für Leser und Rezipienten, aber auch schon für ihre Verfasser und Verbreiter, darstellen, ist unbestreitbar. Dies kann in Unles- und Unschreibbarkeit resultieren, die sich aber fast zwangsläufig ergibt, sobald Lebens- und Lese- bzw. Schreibzeit konfligieren, und wann täten sie dies nicht? Vita brevis, ars longa bzw. 'O $\mu$ غ̀े $\beta$ íos $\beta \rho \alpha \chi \hat{\varsigma} \varsigma$, $\dot{\eta}$ $\delta \grave{\varepsilon} \tau \dot{\varepsilon} \chi \nu \eta \mu \alpha \kappa \rho \alpha ́$ soll Hippokrates das Problem gefasst haben, zumindest war es offenbar schon in der Antike geläufig. ${ }^{1}$ Dass ars und $\tau \dot{\varepsilon} \chi \nu \eta$ dabei weit mehr bedeuten und mehr umfassen als Bücher oder mehrteilige (literarische) Texte oder auch bestimmte große Formen und dass wir grundsätzlich nie auslernen, ist evident. Gleichwohl ist die Problematik allzu langer, allzu umfangreicher und schlicht allzu großer Texte und Bücher - dass also große Formen zu ,übergroßen' werden können - seit dieser Zeit geläufig. Kallimachos

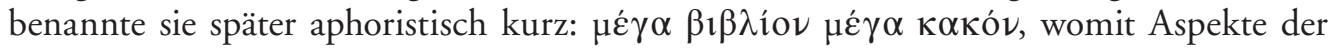
Grenzen von Kunst, Technik und Kunstfertigkeit im Unterschied zum Corpus Hippokratikum außen vorgelassen werden und stattdessen ein medialer und mediologischer Bereich adressiert wird; (allzu) große und umfangreiche Bücher wären demnach bereits alleine wegen ihres Umfangs von Übel, ganz gleich, was sie enthalten mögen, egal auch, ob man sie zu schreiben, zu verbreiten oder zu lesen hat. ${ }^{2}$

Im Folgenden geht es aber nicht allein um die Herausforderungen, die bestimmte literarische Texte (oder auch Kunstwerke allgemein) qua (Über-)Größe an einzelne Leser, Schreiber oder auch andere Personengruppen stellen und stellen können. Denn was heißt überhaupt schon groß, was zu groß? Dass es aufgrund serieller Reihung immer ein Zuviel an Text, Umfang, Quantität und daraus resultierend ein Zuwenig an verfügbarer Aufmerksamkeit, Zeit und Muße geben muss, gehört zur fortwährenden Tragik eines jeden Lesers und zur (dé)formation professionnelle sämtlicher Literaturwissenschaftler, Philologen, Lektoren und Berufsleser. Die Auswege aus dem Dilemma sind bekannt und oftmals unbefriedigend: Noch mehr und pausenlos bis hin zu Schlaflosigkeit und Verzicht auf alles andere zu lesen oder aber es gänzlich einzustellen und einfach sein zu lassen; Lektüren immer stärker kanonisch zu verdichten oder quantitativ auszuweiten, methodisch zwischen close oder deep und distant oder scalable reading, micro, meso und macro analysis hin und her zu pendeln bzw. auf den Differenzen zu bestehen, die sich aus solchen Positionierungen ergeben. Wenn der

1 Hipp. aph. 1,1. Vgl. die lateinische Formulierung zu Beginn von SEnECas De brevitate vitae: „clarorum quoque virorum hic affectus querellas evocavit. Inde illa maximi medicorum exclamatio est: vitam brevem esse, longam artem." (Sen. min. brev. vit. 1,1)

2 Kall. frag. 465 (Pfeiffer)/frag. 511 (Asper); magnus liber, magnum malum (apud Athen. 3,1). 
ideale Leser von Joyces Finnegans Wake (oder auch schon des Ulysses?), aber wohl ebenso von Prousts $A$ la recherche du temps perdu, Musils Mann ohne Eigenschaften, Arno Schmidts Zettel's Traum oder vieler anderer Langtexte unter Schlaflosigkeit leiden müsse, wie Joyce dies in seinem Roman selbst ansprach und damit ein Modell des idealen Lesers moderner Literatur vorgab, ${ }^{3}$ oder aber man die Entscheidung zu treffen habe, schlicht gar nichts mehr zu lesen, was über Metatexte wie Katalogeinträge hinausgeht, wie ein Bibliothekar bei Musil den Ausweg aus dem Dilemma formuliert, ${ }^{4}$ wird deutlich: Das eingangs angesprochene Problem von Lebens- und Lesezeit ist durch Lesen wie Nichtlesen kaum lösbar. ${ }^{5}$

Lebens- und Lesezeit bleiben problematisch aufeinander bezogen, selbst wenn alle Lebens- in Lesezeit aufginge; die entsprechenden Berechnungen führen immer nur vor, dass spätestens seit dem 17. Jahrhundert eine Jahresproduktion an Büchern auch nur einer einzigen Sprache oder Literatur durch schiere Lektüre allein in einer menschlichen Lebensspanne nicht (mehr) zu bewältigen ist. ${ }^{6} \mathrm{Ob}$ dann noch der eine oder andere Romanzyklus, Roman fleuve oder auch nur Großroman hinzukommt oder gleich ganz wegbleibt, ob man Lohensteins (unvollendeten) Arminius-Roman, Balzacs (unvollendete) Comédie humaine oder Musils (unvollendeten) Mann ohne Eigenschaften oder die (teils unvollendeten) Romane Roberto Bolaños vom Espiritu de la ciencia-ficción bis hin zu 2666 gelesen hat, einmal oder gar mehrmals oder auch keinmal, macht vor der erdrückenden Fülle des Ungelesenen keinen so großen Unterschied mehr. Zumal die besonders langen und umfangreichen Großformen oder Übergrößen offensichtlich dazu tendieren, bereits von ihren Verfassern nicht mehr bewältigt werden zu können und echte Fragmente zu bleiben oder nur verkürzt, verändert und verstümmelt ediert und verlegt, zu Betriebsunfall oder intendiertem Programm zu werden; vita brevis, ars longa.

3 Vgl. ,that ideal reader suffering from an ideal insomnia“" (JoYCE [1939, 120] und die Anschlüsse daran zum idealen Modelleser bei Umberto ECO (etwa in I limiti dell' interpretazione, ECO [1990, 106] oder in den HarvardNorton Lectures 1992/93, Eco [1994, 16, 110]).

4 „Aber ich will eigentlich nichts mehr lesen“, äußert Stumm von Bordwehr resigniert, nachdem er vom Bibliotheksdiener Folgendes vernommen hat: „,Herr General,' sagt er ,Sie wollen wissen, wieso ich jedes Buch kenne? Das kann ich Ihnen nun allerdings sagen: Weil ich keines lese!' [...] Es ist das Geheimnis aller guten Bibliothekare, $\mathrm{da} ß$ sie von der ihnen anvertrauten Literatur niemals mehr als die Büchertitel und das Inhaltsverzeichnis lesen. ,Wer sich auf den Inhalt einläßt, ist als Bibliothekar verloren!' hat er mich belehrt." (Musil [1930, 742, 738]).

5 Vgl. zum Lesen, Wiederholungslesen und Nichtmehrlesen auch Weinrich (1984), MoRetTi (Januar/Februar 2000), Bayard (2007) sowie Metz (2008), speziell Moretti zum Problem eines „Great Unread“.

6 Eine der diesbezüglich schönsten Berechnungen, wo das Problem ernsthaft benannt und wiederum verbissen durch Lektüre zu lösen versucht wird: „Das Leben ist so kurz! Selbst wenn Sie ein Bücherfresser sind, und nur fünf Tage brauchen, um ein Buch zweimal zu lesen, schaffen Sie im Jahre nur 70. Und für die fünfundvierzig Jahre, von Fünfzehn bis Sechzig, die man aufnahmefähig ist, ergibt das 3.150 Bände: die wollen sorgfältigst ausgewählt sein!“ (Arno SCHмidT [1988, 30-31]) Vgl. zudem: „Es gibt noch weit beunruhigendere Betrachtungen hier! Setzen wir, daß man vom 5000. Tage an leidlich mit Verstand zu lesen fähig sei; dann hätte man, bei einem green old age von 20000, demnach rund 15000 Lesetage zur Verfügung. Nun kommt es natürlich ebenso auf das betreffende Buch, wie auch auf die literarische Aufnahmefähigkeit an. [...] Sagen wir, durchschnittlich alle 5 Tage 1 neues Buch - dann ergibt sich der erschreckende Umstand, daß man im Laufe des Lebens nur 3000 Bücher zu lesen vermag! Und selbst, wenn man nur 3 Tage für eines benötigte, wären's immer erst arme 5000. Da sollte es doch wahrlich, bei Erwägung der Tatsache, daß es bereits zwischen 10 und 20 Millionen verschiedener Bücher auf unserem Erdrund gibt, sorgfältig auswählen heißen. [...] Sie schaffen in Ihrem Leben nicht einmal sämtliche Bände der Hochliteratur!" (SCHMidT [1995, 91-92]) 
II. Edieren als defizitäre Praxis. Unvollendete, unvollendbare Romane, die letztlich unschreibbar und unlesbar bleiben - neben Produktion und Rezeption weist die große Form und das daraus resultierende dicke Buch auch eine editorische Komponente auf, die hier reflektiert werden soll.7 Sie ist eng verknüpft mit der Unschreibbarkeit und Unlesbarkeit großer Formen bzw. ein Resultat des eingangs angeführten Dilemmas: Unvollendete Texte müssen nicht nur gelesen und verstanden, sondern mitunter auch weitergeschrieben werden, sollen sie nicht für immer unvollendet und unlesbar bleiben. So lautete zumindest bis weit ins 20. Jahrhundert hinein die editorische und textkritische Überzeugung, die sich mit echten Fragmenten nur ungern zufrieden gab und editorische Entscheidungen traf und Texteingriffe vornahm, wie sie in den letzten Jahrzehnten vielfach als unzulässig gelten müssen. Mit Fausts Famulus Wagner, von dem die bekannteste deutschsprachige Fassung des vita brevis, ars longa-Diktums stammt, haben wir nicht nur einen am Verfassen von Reden rhetorisch interessierten Leser vor uns, sondern auch jemanden, der editionstheoretische Forderungen (Stichwort, Quellenkritik' und in primis ad fontes) in den Blick nimmt bzw. zumindest antönen lässt. Im Faust von 1790 heißt es:

\author{
Ach Gott! die Kunst ist lang; \\ Und kurz ist unser Leben. \\ Mir wird, bey meinem kritischen Bestreben, \\ Doch oft um Kopf und Busen bang'. \\ Wie schwer sind nicht die Mittel zu erwerben,
}

7 Editorik verstanden als textwissenschaftliche und philologische Methode und Disziplin, aber auch als Praxis. Es wäre spannend, der Frage nach der Verbreitbarkeit und Verlegbarkeit, aber auch Verkäuflichkeit und grundsätzlichen Marktgängigkeit großer Formen und umfangreicher Buchpublikationen gesondert nachzugehen, was vom Fortsetzungs- und Serienroman bis hin dazu reichen kann, zu welchen Zeiten dicke Romane gewagt werden (und gefragt sind - und wann nicht), wann sie ,drehen' (und wann unter keinen Umständen) und wie es mit ihrer Verfügbarkeit im stationären Buchhandel aussieht. Seit der Moderne oder zumindest im 19./20. Jahrhundert gilt die kleine Form als bevorzugt rezipierbare. Einschlägige (theoretische) Ausführungen und Behauptungen dazu reichen von Poe über Huysmans zu Lewis Carroll. Gelungen ist Alfred Polgars Formulierung von 1926: „Das Leben ist zu kurz für lange Literatur, zu flüchtig für verweilendes Schildern und Betrachten, zu psychopathisch für Psychologie, zu romanhaft für Romane, zu rasch verfallen der Gärung und Zersetzung, als daß es sich in langen und breiten Büchern lang und breit bewahren ließe. Daß die Schriftsteller Zeit finden, weitläufig zu schreiben, kann ich zur Not verstehen: [...] Aber daß Menschen dieser tobenden, von nie erlittenen Wehen geschüttelten Epoche Ruhe und Zeit, innere Zeit, finden, weitläufig zu lesen, ist mir ein rechtes Mirakel. [...] Ewigkeiten erweisen sich als zeitlich, die solidesten Götter als Götzen, alle Anker sind gelichtet, kein Mensch weiß, wohin die Reise geht, aber daß sie geht und wie sausend rasch sie geht, spüren wir am Schwindel: wer wollte da mit überflüssigem Gepäck beladen sein? Ballast ist auszuwerfen - und was entpuppt sich nicht als Ballast? -, kürzeste Linie von Punkt zu Punkt heißt das Gebot der fliehenden Stunde. Auch das ästhetische. ,Schöne Literatur' mit geschwollnem Wanst ist ein Widerspruch im Beiwort.“ (Polgar [1984, 372-373]) Das verhindert aber nicht seither wiederkehrende Wellen umfangreicher und dicker Bücher - nicht erst zum lateinamerikanischen Boom der 1970er oder dem Romanschaffen nach 2000, vgl. schon Musils Klage über seinen "Zufalls- und Interimsverleger Dr. Menzel“ anlässlich der Publikation von Nachlass zu Lebzeiten im Zürcher Humanitas-Verlag, als er zeitgleich seinen Mann obne Eigenschaften nicht abschließen konnte, aber dringend Geld benötigte: „Sehr dicke Bücher sollen jetzt in Amerika wieder modern sein. Ursache, wie anzunehmen ist, irgendein grosser Erfolg eines Wälzers. Die Augen Dr. Menzels (Humanitas Verl.) leuchten sachverständig auf, wenn er mir erzählt, dass von kurzen Geschichten (Nachlass) jetzt in Amerika nichts zu erwarten wäre. Ein neues Buch von ihm, das höchstens 400 normale Seiten hätte, ist auf 675 gebracht und dann noch durch dickes Papier und Einband so gestaltet, dass es fast ein Würfel, und so dick wie hoch ist. Schon die Augen sehen: das konfektionelle Extrem einer Mode." (Musil [2009, Transkriptionen/Mappe VIII/5/43]). 
Durch die man zu den Quellen steigt!

Und eh' man nur den halben Weg erreicht,

Muß wohl ein armer Teufel sterben. ${ }^{8}$

Doch selbst, wenn die Lebenszeit ausreicht, wenn eine große Edition nach oft jahre- oder jahrzehntelanger (auch arbeitsteiliger) Arbeit mehr oder weniger fertig vorliegt, ist damit noch nicht alles gelungen und das Wesentliche am Ende vielleicht unerfüllt geblieben. Kein Editor gesteht es sich gerne ein, dennoch zeigt jede Edition immer auch das Scheitern vorgängiger Editionspläne und -absichten. ${ }^{9}$ Was zu Beginn einmal denk- und wünschbar war oder gewesen wäre, wird schließlich allen möglichen Zwängen geopfert und findet selten den Weg auf Druckseiten oder in elektronische Formate. Ideal und Wirklichkeit decken sich kaum, doch bei Editionen wohl nie. Es gibt eine Unedierbarkeit der Welt und eine auch der Gegenstände einer Edition. Die edierten Objekte sind niemals die Objekte selbst; es bleibt eine ontologische Distanz, die fortwährend schmerzhaft und demütigend ist. Im Bereich der Textwissenschaften, wo dieser Abstand im autographen und allographen Bereich besonders evident ist, edieren wir keine Originale und nie die Werke selbst, sondern letztlich immer nur Derivate: Abschriften, Kopien, Transkriptionen, Transliterationen von etwas Vorgängigem. Edieren ist nicht nur sammeln, bewerten, gewichten, aussondern, emendieren und verbessern, sondern fast zwangsläufig ausschließen, reduzieren, verzichten, verlieren, sogar vernichten. Die Objekte oder Dinge selbst - ad fontes, „zu den Quellen“ ist der trügerische Wunsch und die unerfüllbare Verheißung Wagners - stellt keine Edition je wirklich vor Augen. Keine kann je deren Realpräsenz (wieder)erschaffen, auch keinen unvermittelten und direkten Zugang zu ihnen. ${ }^{10}$

8 Goethe (1790), 15; vgl. Goethe (2018).

9 Und vielleicht auch das Scheitern und die Erschöpfung eines Editorenlebens. So stellte sich Bernhard Suphan kurz nach Erreichen seiner Pensionierung als erster Direktor des Goethe-Schiller-Archivs und Mitverantwortlicher der Weimarer Goethe-Ausgabe (Sophienausgabe) auf einen Stapel seiner bis heute als Referenz geltenden 33-bändigen Ausgabe von Herders Sämmtlichen Werken und erhängte sich kurz nach seinem 66. Geburtstag. Wenig später erschien ein zehn Jahre früher verfasster Text, im Goethe-Jahrbuch als Miszelle vom Herausgeber mitgeteilt: „, Ardua res est vetustis novitatem dare, obsoletis nitorem.< Dies Wort des älteren Plinius steht als Motto vor dem dritten Teile von Herders Ideen zur Philosophie der Geschichte der Menschheit. ,Ein schweres Stück Arbeit ist es, Altertümlichem den Reiz der Neuheit zu geben, Veraltetes in neue Beleuchtung zu stellen.، Ich habe das mit Herder selbst versucht, es konnte nur gelingen, indem man entschlossen den geschichtlichen Standpunkt annahm und dem Leser anwies, indem man zeigte oder empfinden ließ, wie frisch und triebkräftig das Meiste gewesen, als es in Erscheinung und Wirkung trat. Hätte ich mir denken können, welche Schwierigkeiten, welche 'Steilen ( hätte, vor drei und dreißig Jahren. Gut, daß ich es nicht gedacht, nicht bedacht habe. >Man wird Ihnen den letzten Band auf den Sarg legenı, hat mir einmal, in dem ersten Stadium der Arbeit, Theodor Mommsen gesagt, auch in diesem Falle ein Wissender. Das Wort war nicht gesprochen, um mich zu schrecken. Doch ist manchmal, ich gestehe es, eine Scheu über mich gekommen, den letzten Band herauszugeben. Nun ist, dank der Beihilfe eines treuen, vortrefflichen Freundes, auch dieser der Vollendung nahe [...,] er trägt die Zahl 33. Soll es einmal in einem Nachrufe heißen: B. S. Herderum edidit. Obiit. Hat den Herder herausgegeben, danach das Zeitliche gesegnet, mir solls recht sein. [...]' Wie prophetisch sind Mommsens Worte gewesen, die letzten Bände sind Suphan wirklich mit in den Sarg gelegt worden." (GeIGER $[1912,232])$

10 Vgl. dazu im Vorwort zur neuen Essaysammlung von SHILLINGSBURG (2017a, viii): „[I]t should be noted that no one ever read the original of any literary work; one can only read a copy - manuscript, proof, or print - each is a copy or a copy of a copy. None, not even the first copy, is the original. We work at an unbridgeable distance from the object of our attention. Is it a book? Is it a text? Is it a work? What are the consequences of that gap?" 
Zu dieser deprimierenden Behauptung bzw. diesem resignativen Befund kann man sich verschieden verhalten, melancholisch, wütend, gleichgültig, ablehnend. Andere Reaktionen sind ebenfalls denkbar. Und doch bleibt ein Stachel oder zumindest ein Unbehagen: Egal wie ausgesucht und passend die zugrundeliegenden Editionsverfahren und technischen Umsetzungen auch ausfallen, die Schönheit, Intaktheit und ursprüngliche Verfasstheit der Gegenstände selbst, die ediert werden, ist immer eine andere als das, was sie in edierter Form letztlich noch darzustellen vermögen: als Residuum, Reminiszenz, Spur, Substrat, Überbleibsel. Aus einem Textabschnitt, einer Scherbe, einem Papyrosrest eine ganze vergangene Welt, Kultur, Kunst oder zumindest ein Werk wiederentstehen zu lassen - dieses Versprechen eines an sich magischen Prinzips, über ein magnum in parvo immer wieder Zugang und Unmittelbarkeit neu entstehen zu lassen, wird konfrontiert mit der Verlustgeschichte, die eine jede historische Entwicklung und mediale Übertragung zwangsläufig mit sich bringt.

Zugleich ermöglichen Editionen epistemische Zugewinne und erschaffen, was es zuvor nicht gab und ohne sie so kaum geben kann: Abstraktion, Auffindbarkeit, Sichtbarkeit, Lesbarkeit, Wissen, Kontexte und Zusammenhänge. ${ }^{11}$ Sie bewahren und verdauern, rahmen und kontextualisieren, erklären, kommentieren, illuminieren und verbessern, führen in vielen Fällen dazu, dass edierte Gegenstände, in welcher Form schließlich auch immer, vom Code bis zum dreidimensionalen Abbild, in irgendeiner Weise vorhanden sind, aber auch verfügbar werden und auf Dauer existieren. Durch Ausgaben und Editionen kommt es - zumindest im Bereich textueller Objekte - oft genug überhaupt erst zur Realisierung von Kunstwerken, dazu, dass es diese als Kunstwerke überhaupt geben kann. Denn als singuläre und unikale wären sie nicht nur gefährdet, in Vergessenheit zu geraten, sondern auch ohne Publikum. Der ontologische, doch ebenso kulturelle Status von Text-Kunst hängt entsprechend auch davon ab, dass nicht nur geschrieben und produziert, sondern zudem publiziert, herausgegeben, ediert wird. ${ }^{12}$ Editionen verschließen und reduzieren die enormen Möglichkeiten von Werken, erschließen sie aber zugleich und realisieren sie überhaupt erst. ${ }^{13}$

Damit ist - für die allographe Textüberlieferung ohnehin - das Problem bei Editionen seltener, dass es sie gibt und dass sie zu viel tun, etwa mutwillig abändern oder nichtneutral operieren, sondern oftmals, dass es sie nicht gibt und dass es von allem zu wenig gibt. Jede Edition bewahrt und konserviert mehr, als sie verstellt und zerstört. Eine Textüberlieferung ohne editorische und publikatorische Transmission, wie auch immer sie später qualitativ eingeordnet werden sollte, ist undenkbar. ${ }^{14}$ „Drum besser wär’s daß nichts entstünde“,

11 Vgl. zu neugermanistischen Editionen und deren Editoren KamzelaK, Nutt-Kofoth, Plachta (2011).

12 Vgl. zum kulturellen Status von Ausgaben sämtlicher Werke auch CAHN (2004).

13 Vgl. dazu den Beginn von Shillingsburgs How Literary Works Exist, ein erstmals 2007 am University College London vorgestellter Vortrag: „Literary works are not known without media; they are known only by means of copies. There is no original of which all other exemplars are copies; the original is itself just the first copy. What are the copies of? The work. It is like a mystery: No copy is the work; every copy is the work. Each copy represents the work; no copy represents the work fully. Documents and texts exist as material objects, copies. Works, on the other hand, come into and go out of existence as mind objects, engagements with copies. Textual scholars care for material objects because they are the necessary media for the mind objects we all care about. [...] It is not a mystery; it is a subject for analysis and debate." (SHILLINGSBuRG [2017b, 115])

14 Immer noch (und immer wieder) lesenswert dazu sind die Beiträge der beiden Bände der Geschichte der Textüberlieferung, auch noch nach fast 60 Jahren; ein Beispiel, dass philologisches Arbeiten zu dem zählen kann, was bleibt: Hunger u. a. (1961); LANGOSCH u. a. (1964). 
behauptet eine weitere Faust-Figur, und Mephistopheles mag recht damit haben; aber wohl kaum damit, dass es besser wäre, wenn nichts ediert würde, nachdem es entstanden ist. ${ }^{15}$ Die Crux zwischen Zuviel und Zuwenig besteht gerade darin, dass es keine Autographen antiker Autoren gibt und auch die Überlieferungslage von Texten erst da passabel wird, wo sie nicht mehr so dringlich benötigt wird und es dann sogar ein Zuviel geben kann. ${ }^{16}$

III. Unmögliche Editionstypen. Im Weiteren soll es darum gehen, dass Editionen aufgrund ihres Umfangs und quantitativer Aspekte, aber auch der Zeit, die es zu ihrer Verfertigung und schließlichen Lektüre braucht, vielfach Unmögliches berühren oder gar selbst unmöglich werden. Dies hat allerdings auch einen praxeologischen und rezeptionstheoretischen Hintergrund. Nicht jede existierende Edition wird adäquat oder ihrer Ausrichtung gemäß genutzt, und schon gar nicht jede auch gelesen. Editionen produzieren mitunter, je näher sie dem Ideal einer totalen Edition kommen, akribisch alles möglichst verlustfrei zu edieren, bestimmte Abwehrhaltungen auf der Seite prospektiver Nutzer. Sie sind dann - je perfekter und sorgfältiger sie ausfallen, umso stärker, was paradox ist - mit dem Vorbehalt belastet, ihre Gegenstände geradezu zu verstellen, zu verbergen, unzugänglich, unlesbar zu machen und Schwellen zu errichten, die nicht nur durch hohe Preise, Lizenzabgaben oder Paywalls errichtet werden. Das klingt konstruierter, als es im täglichen Umgang mit ihnen tatsächlich ist.

Denn ein Einwand gegen wissenschaftliche und entsprechend komplexe und aufwendige kritische oder historisch-historische Editionen lautet, sie zu nutzen sei nicht sie zu lesen, ihr Studium nicht gleichbedeutend mit ihrer Lektüre. Wobei wir Bücher - und gedruckte Editionen sind bis heute meist in Buchform organisiert, nicht allein textbasierte, sondern etwa auch musikalische oder solche aus anderen Bereichen - nicht notwendig linear und von der ersten bis zur letzten Seite lesen; wohl aber mit einer Wahrnehmung für deren Umfang und die Reihenfolge der edierten Materialien. Umfang strukturiert Inhalte, schreckt dabei aber wohl oft genug auch von einer Annäherung ab, sobald Werkausgaben in mehreren Regalmetern daherkommen. Bei digitalen Ausgaben wird diese Form des Überblicks erschwert, zugleich bieten sie gänzlich andere Rechercheoptionen. Den

15 Goethe $(1808,87)$. Dennoch verstellen schlechte Editionen, was es hätte geben können, zementieren schlechte Lektüren, Lesarten, Darbietungsformen. Zugleich zeigt selbst ein so extremer Fall wie Max Brods Eingriffe in Kafkas Texte, dass sogar damit noch der Nachlass und die Rezeption gesichert und bewahrt werden konnten, also auch noch die schlechteste Edition mehr Nutzen als Schaden bewirken kann.

16 Vgl. „Von den bedeutsamsten Denkmälern fehlen uns meistens die Originale [...]. Dafür besitzen wir von viel kunstärmerer Dichtung öfter zu viele Handschriften, so von dem eigentlich unlesbaren Kalendergedicht des Hugo von Trimberg, der Laurea sanctorum, 19, von moralischer Schuldichtung, wie dem Liber quinque clavium und den Arbores virtutum et viciorum, über 30, von der antiklerikalen Satire der Apokalypse des Golias 68, von den Streitgedichten der Visio Philiberti und der Ecloga Theoduli 150 bzw. etwa 200 Handschriften und rund 100 Drucke. [...] Im ganzen ergibt sich, daß das Mittellatein mehr das Schulmäßige als das Wertvolle tradierte - und damit steht es keineswegs allein." (LANGOSCH [1964, 24]) - Bücherflut auch für Leser; nicht nur für Editoren, die das alles unmöglich bewältigen können. So heißt es weiter: „Die Überlieferungsgeschichte ist beim Original insofern einfacher gelagert, als für sie nur wichtig ist, über welche Orte es durch die Jahrhunderte weitergereicht wurde, wo, wann und wie man es abschrieb und benutzte. Sie ist aber bei der übrigen Masse des Überlieferten, von dem nur mehrere nicht originale Handschriften existieren oder gar nur eine solche, durch die zu leistende Editio critica umfangreicher, schwerer und unsicherer." (ebd.) 
Überblick wiederzuerlangen, den eine Druckausgabe durch die topologische Zuordnung von Inhalten zu einer bestimmten Aufteilung auf Bände, Seiten und dadurch zu Zahlen und Positionen ermöglicht, ist wünschenswert. Digitale Editionen - auch solche, bei denen es keine gedruckten Anteile mehr gibt - benötigen dies ebenfalls.

Dass wir Bücher lesen, nicht Editionen, und Lesen nur eine mögliche Nutzungsform von Büchern darstellt, ist relevant, weil Bücher nicht einfach neutrale Medien sind, sondern Inhalte maßgeblich konfigurieren und prägen. ${ }^{17}$ Bücher wollen gelesen sein, um verstanden zu werden, was wiederum Hybrideditionen im Verbund mit gedruckten Texten eine besondere Rolle zukommen lässt. Und dafür spricht, Kommentare und Apparate auszulagern und Lesetexte zu konstituieren, die einfach auch nur zur Lektüre konsultiert werden dürfen, ohne dass die Lesenden sich zuvor mit einem komplexen Apparat auseinanderzusetzen hätten, der dieses Lektüreerlebnis und diesen einfachen Zugang verstellt. Hybridausgaben versuchen eine mehrfache Adressierung: Interessierte oder einfache Leser sollen genauso berücksichtigt werden wie philologisch oder wissenschaftlich orientierte Nutzer, wobei beides in Personalunion auftauchen kann.

Die Versöhnung von Lesern und Nutzern ist freilich ein altes Anliegen und geheimer Traum der allermeisten Editoren, ganz gleich, wie ihre Editionen ausfallen, und egal, welchen Ausgabentypus sie bedienen. Ein Leserbrief, den kein geringerer als Richard Alewyn, selbst bedeutender Hofmannsthal-Forscher, 1974 mit Bezug auf den Beginn der kritischen Hofmannsthal-Ausgabe schrieb, ${ }^{18}$ zeigt komprimiert auf, dass (historisch-)kritische Ausgaben oft genug nicht gelesen werden (können) und damit eine Form unmöglicher Edition darstellen. So schrieb Alewyn anlässlich der kurz zuvor in der Frankfurter Allgemeinen Zeitung vorgestellten und damals noch bandreicher angelegten Hofmannsthal-Ausgabe ${ }^{19}$, was als Einwand eines maximal gebildeten und einschlägig informierten Lesers („Mir ist die Kompliziertheit von Hofmannsthals Nachlaß nicht unbekannt.“) erstaunlich ist:

Es ist erfreulich, von Dr. Lüders zu hören (F.A.Z. vom 15. Februar), daß die ersten Bände der Hofmannsthal-Ausgabe im nächsten Jahr erscheinen werden, besonders für jemanden wie [m]ich, der diese Botschaft schon vor 28 Jahren zum ersten Mal gehört hat, wenn auch aus anderem Munde. [...] Aber Dr. Lüders bekennt gleichzeitig, daß diese ersten Bände nicht für jedermann bestimmt sind. Sie sind die Anfänge einer auf fünfzig Bände veranschlagten historisch-kritischen Ausgabe, deren Abschluß nur die Jüngeren von uns erleben werden und die dazu bestimmt sein wird, in Universitätsbibliotheken eingesargt zu werden. ${ }^{20}$

Alewyn begründet dies durch die Gegenüberstellung von zwei Nutzungsweisen und die Behauptung, kritische Ausgaben würden ohnehin nie gelesen, sondern immer nur benutzt werden. Benutzt, nicht einmal genutzt, eine arge stilistische Verschärfung: „Aber eine solche Ausgabe ,benutzt' man, man liest sie nicht. (Wer hätte wohl den ,Urfaust' zuerst in der Weimarer Ausgabe gelesen?) Hofmannsthal aber hat für Leser geschrieben, nicht für

17 Vgl. dazu auch Metz (i. Dr., xx).

18 Hofmannsthal (1975-202X).

19 In den nächsten Jahren wird sie mit Erscheinen von Band 42 abgeschlossen werden.

20 Alewyn (23./24.2.1974, 24); vgl. zudem Alewyn (15./16.5.1976). 
Benutzer, Leser auf der Couch, am Badestrand". ${ }^{21}$ Die kritische Hofmannsthal-Ausgabe sei nur benutzbar, aber nicht lesbar, gehöre in den Lesesaal einer Bibliothek, aber nicht auf die Couch im heimischen Wohnzimmer oder ein Strandtuch im Freien; als Korrektiv wird statt der „vom Freien Deutschen Hochstift vorbereiteten Monumentalausgabe“, wie Alewyn sie nennt, vorgeschlagen, „,in ein, zwei Jahren in drei, vier Bänden die bedeutendsten der unbekannten Nachlaßfragmente in einer wohlfeilen Ausgabe zusammenzustellen." Denn nur eine solche wäre „auch für den durchschnittlichen, auf nichts als Genuß erpichten Leser erschwinglich“ und leicht fertigzustellen gewesen: „Ein einziger Mann dagegen hätte ausgereicht," so aber werde „durch ein Aufgebot von Dutzenden von Köchen, die in aller Welt verstreut sind“, alles verdorben und kompliziert. Abschließend macht Alewyn erstaunliche philologische Zugeständnisse: „Auch wenn dann hier oder dort ein Komma an der falschen Stelle säße oder ein Krümelchen fehlte, es wäre damit Hofmannsthal ein Dienst getan und seinen Lesern eine Freude gemacht."

So ungerecht vieles an Alewyns Darstellung sein mag, nicht zuletzt, weil kritische Ausgaben später häufig als günstige Studienausgaben in ihren Lesetexten nachgedruckt werden, trifft seine Gegenüberstellung von Lesern und Nutzern doch einen empfindlichen Punkt. Editionen müssen sich meist auf eine der beiden Seiten schlagen, dabei aber die jeweils andere vernachlässigen. Zwischen bequemer Nutzung (,auch für den durchschnittlichen, auf nichts als Genuß erpichten Leser erschwinglich“, dem dadurch „eine Freude gemacht" würde) und philologischer Maximalverpflichtung („ein Komma [...] oder ein Krümelchen") ist nicht gut zu vermitteln. ${ }^{22}$ Auch die in Klammern gesetzte rhetorische Frage Alewyns trifft: „(Wer hätte wohl den ,Urfaust' zuerst in der Weimarer Ausgabe gelesen?) “. ${ }^{23}$ Dabei fragt er nach dem Urfaust, nicht nach dem Faust, und damit nach einem philologisch gesehen gar nicht ganz so unproblematischen und deutlich komplexeren Gegenstand als etwa einem vom Verfasser autorisierten und publizierten Text wie dem Faust-Druck von 1790. ${ }^{24}$

21 Hier und im Folgenden in diesem Absatz: Alewyn (23./24.2.1974, 24).

22 Zum Thema Nutzerkomfort noch einmal Shillingsburg, wenn er schreibt: „Convenience can hardly be overemphasized. [...] Convenience is, in practice, one the most important qualities of a text. All else being equal, students and critics probably care about the quality of texts. Given an equal choice, none would say ,I prefer an inaccurate text of unknown origin.' Given easy access to contextual information about the texts being studied, few would say ,I really do not want to know.' In practice, however, many proceed without knowing because it is convenient. [...] Hence, most literary criticism is conducted in the presence of convenient, cheap editions, often paperbacks, with texts that can be dog-eared, underlined, written in, or even torn apart. Unfortunately, such texts often lack accurate acknowledgements of sources and have no trace of original fonts, formats, or forms, or even descriptions of those forms. Hence, much literary criticism is based on texts with dubious claims to textual accuracy, without textual histories, and often without historical contexts. Why are these the tools of choice for literary criticism? They are convenient. [...] Scholarly editions have not been convenient because textual criticism is complex [...]. Perhaps students and critics willing to forego knowledge have no idea how much they are paying for their convenience. Reforming them is not our job; making knowledge accessible and convenient may be." (Shillingsburg [2017c, 140-141])

23 Alewyn (23./24.2.1974, 24).

24 Der Urfaust, auch dieser Titel ist eine editorische Setzung, wurde 1887 von Erich Schmidt im in Dresden befindlichen Nachlass von Luise von Göchhausen - die 1775 eine Vorlesefassung Goethes abgeschrieben hatte - entdeckt und ediert, wenn auch mit erheblichen Eingriffen in das Manuskript: Goethe (1887a). Schmidt verantwortete aber auch die zeitgleich erschienenen Faust-Bände der Weimarer Ausgabe (GoETHE (1887b, 1888a und 1888b). An seiner editorischen Einrichtung kam also keine nachfolgende Ausgabe des Urfaust vorbei, und gut zwei Jahrzehnte war der Urfaust daher wirklich nur „zuerst in der Weimarer Ausgabe“ zu lesen. Einfach 
Es stellt sich die Frage, was eine Edition leisten muss, um nicht nur Nutzer, sondern auch Leser zu erreichen. Attraktiver Preis und Publikumsverlag sind sicher von Vorteil, eine gelungene Buchgestaltung mag ebenso von Belang sein. Alewyn spricht mit seiner „in ein, zwei Jahren [...] zusammenzustellen[den]“ „wohlfeilen Ausgabe“ daneben eine zeitliche Dimension an, die wieder das vita brevis, ars longa-Thema berührt: ein nicht wesentlicher Makel der neuen Hofmannsthal-Ausgabe, die bis heute nicht fertiggestellt ist, sei ihre auf Jahre und Jahrzehnte hin angelegte Erscheinungsdauer (wie die einer jeden kritischen oder historisch-kritischen Großedition), so dass „deren Abschluß nur die Jüngeren von uns erleben werden“. ${ }^{25}$ Denn auch eine Ausgabe, deren Erscheinungsverlauf die eigene Lebensspanne überschreitet, ist letztlich im individuellen Fall unlesbar. Sie läuft Gefahr, aufgrund der Kontingenz von Finanzierungszusagen, der Lebensdauern von Editoren oder von Verlagen und vielem anderen, nicht plangemäß abgeschlossen werden zu können.

IV. Unmögliche Editionsgegenstände. Fragen nach der Edierbarkeit großer Formen kommen schließlich auch da auf, wo eine solche grundsätzlich endet und unmöglich wird, jenseits finanzieller oder zeitlicher Einschränkungen und auch jenseits einer späteren Rezipierbarkeit bzw. praktischen Verwendbarkeit vorliegender Editionen. Etwa, weil die zu edierenden Gegenstände gänzlich unedierbar sind. Die Grundüberzeugung eines jeden Editors wäre freilich erst einmal - von prinzipiellen Vorbehalten wie den in der zweiten Sektion oben bereits geäußerten abgesehen -, dass sich schlicht alles Symbol- und Zeichenhafte in irgendeiner Weise edieren lässt. Zumindest alles, was nicht verlorengegangen ist, zerstört wurde oder aus anderen Gründen nicht mehr zu rekonstruieren ist und daher nicht mehr zur Verfügung steht. Doch stimmt das denn? Gibt es nicht auch Editionsgegenstände, die sich zur Gänze entziehen, unmögliche Editionsprojekte, deren Scheitern unabdingbar und notwendig ist? Im Akzente-Themenheft Unmögliches führen Clemens J. Setz und Jo Lendle 2015 zwei monströse Schreibprojekte an, die dort als unedierbar deklariert werden und eine eigene Taxonomie definieren, das Voynich-Manuskript sowie Henry Dargers Book of Weather Reports:

Über das berühmte Voynich-Manuskript, das 1912 von einem Buchhändler erworben wurde und das bislang weder von Wissenschaftlern noch von Computern entziffert werden konnte, [...] wird [...] diskutiert, ob die Herstellung eines solchen Buches leicht oder schwierig ist. Manche Wissenschaftler, vor allem Mathematiker, halten es für sehr einfach. Anderen kommt es schlichtweg unmöglich vor.

Das unmögliche Buch oder die Unmöglichkeit an sich hat viele Menschen beschäftigt. Henry Darger, der exzentrische Autor eines fünfzehntausend Seiten langen graphischen Romans, welcher erst nach seinem Tod in seinem Apartment in Chicago entdeckt wurde, führte jahrzehntelang ein Wetterjournal, in dem er nicht nur festhielt, wie das Wetter an einem bestimmten Tag gewesen war, sondern auch, in welchen Punkten der weather man im Fernsehen sich in seiner

erhältliche reine Textausgaben, auf die sich Alewyn hier bezieht, gab es erst später, in Reclams Universalbibliothek etwa unter Nr. 5273 ab 1911 (GOETHE [1911]), dann aber massenhaft (vgl. für Einzelpublikationen DRIESCH, SCHLAGER [1957-1960, 773]).

25 Alewyn (23./24.2.1974, 24). 
Vorhersage geirrt hatte. [...] Der TV-Meteorologe wird in Dargers Book of Weather Reports zu einer literarischen Figur, tragisch wie Faust, komisch wie Don Quijote. Publizieren lässt sich Dargers Werk nach wie vor nicht, zumindest nicht in seiner Gesamtheit. Es übersteigt die Möglichkeiten herkömmlicher Editionsarbeit. ${ }^{26}$

Nun wird allerdings nicht näher erläutert, worin diese Übersteigerung der „Möglichkeiten herkömmlicher Editionsarbeit" genau besteht. Das Voynich-Manuskript und Dargers Weather Reports (oder seine Story of the Vivian Girls) werden zu Extrembeispielen stilisiert, um im Akzente-Heft ebenfalls näher vorgestellte unmögliche Werke einordnen zu können (etwa den knapp 1500 Seiten umfassenden japanischen Science Fiction-Roman Yapu von Shozo Numa, das Esperantowerk von William Auld, u. a. La infana raso, eine über 1300-seitige Beschreibung von Connemara, Heimatort des irischen Kartographen Tim Robinson, oder auch Goethes Faust, I. und II. Teil, zu dem Robert Menasse Einblicke in seine Lektürebiographie gewährt, „Mein Leben mit Doktor Faust“). Entsprechend heißt es:

Einige der in dieser Ausgabe auszugsweise vorgestellten Werke fallen in die Darger-Kategorie und in einigen Fällen berühren sie sogar die des Voynich-Manuskripts: Bücher, bei denen es zutiefst überrascht, dass sie überhaupt in irgendeiner Sprache, in irgendeiner Form verfasst wurden, zu groß, zu enorm scheint der Widerstand traditionellen Lektüreempfindens. [...] Neben der paradoxen Gruppe dieser existierenden unmöglichen Bücher gibt es die unüberschaubare Menge der tatsächlich unmöglichen Literatur, die ihrem Namen Ehre macht und ungeschrieben bleibt. ${ }^{27}$

Nun ist unbestreitbar, dass Ungeschriebenes und nicht Realisiertes weder ediert noch rezipiert werden kann: Ideen, Entwürfe, Skizzen, die nie eine wie auch immer geartete Spur, keinen Abdruck und keinen Eindruck hinterlassen haben, keinerlei Markierung und Hinterlassenschaft konstituieren, entziehen sich auch grundsätzlich einer Les- und Edierbarkeit. Auf der anderen Seite zeigt die Geschichte der Konzeptkunst, aber auch allein die Arbeit einer Gruppierung wie OuLiPo (Ouvroir de la littérature potentielle), dass noch aus geringsten Angaben, Anleitungen und Beschreibungen sehr komplexe Kunstwerke entstehen können (oder jenseits ihrer Realisierung zumindest könnten) und daneben auch Titel, Plots, Spielregeln oder Fragmente ungeschriebener oder unrealisierter Werke grundsätzlich edierbar sind. ${ }^{28}$ Das gilt schließlich auch für die beiden die Taxonomie von Setz und Lendle aussteuernden Großtexte: Natürlich lässt sich das Voynich-Manuskript edieren, eventuell sogar verstehen, ${ }^{29}$ wie jeder unlesbare oder unentzifferbare andere Text auch, solange er nur

26 Lendle, Setz $(2015,1)$.

27 Lendle, Setz $(2015,2)$.

28 Ein Beispiel dafür wäre die Anthologie von Pehnt, Holder, Staiger (2014), wo 71 Autoren der deutschsprachigen Gegenwartsliteratur von Volker Harry Altwasser bis Juli Zeh Titel ungeschriebener Werke nennen und erläutern, jeweils mit aktuellen Umschlagentwürfen versehen. Oder auch die teilweise niemals intendierte Buchprojekte aufnehmende Sammlung von Mittelmeier (2006), wo unterschiedliche Werkstufen von Goethe bis Jandl vorgestellt werden; sowie die deutlich weniger spielerische Zusammenstellung von BARTL, ERK, KRAUS (2019), die von Lessings Faust-Fragment bis hin zu Philipp K. Dicks Fortsetzungsversuchen zu The Man in the High Castle reicht.

29 Eine rezente Behauptung der Entschlüsselung des seit 1969 in der Beinecke Rare Book and Manuscript Library der Yale University befindlichen Manuskripts stellt CHESHIRE (2019) dar. 
reproduktionsfähig ist,$^{30}$ und selbstverständlich ist die Herausgabe und Edition auch umfangreicher Mixed Media-Arbeiten wie derjenigen Dargers kein grundsätzliches Problem, sobald bestimmte Voraussetzungen vorliegen. ${ }^{31}$ Mit „der paradoxen Gruppe dieser existierenden unmöglichen Bücher" scheint es aus editorischer Sicht unproblematisch zu sein. ${ }^{32}$

Entsprechend gibt es Langzeiteditionsprojekte, die über sehr lange Zeiträume unter Beanspruchung großer personeller und finanzieller Kapazitäten erstellt wurden oder werden,

30 Ein Beispiel wäre Luigi Serafinis Codex Seraphinianus, der mittlerweile in einer Vielzahl von Neuauflagen und sogar fremdsprachigen Ausgaben vorliegt, was seine Entzifferung nicht ermöglicht hat, aber zumindest seiner Verbreitung dienlich war (SERAFINI 1981). Die Frage der (unkritischen) Reproduktion umfasst aber auch die Frage, was schon als Edition zu zählen sei, die durchaus kontrovers ist. So definiert etwa SAHLE $(2017,239)$ : „Edition ist die erschließende Wiedergabe historischer Dokumente. [...],Wiedergabe bezeichnet das notwendige Kriterium der vollständigen Repräsentation des Editionsgegenstandes (merke: Eine Beschreibung, ein Kata$\log$, ist noch keine Edition!) auf bildlicher und/oder (meistens) textlicher Ebene. ,Erschließung 'schließlich ist ein Oberbegriff für alle kritischen Operationen, von der Auswahl der zu edierenden Objekte über die äußere Beschreibung, die selbst in einer Transkription unvermeidlichen Entscheidungen, die Identifikation von referenzierten ,Dingen' in Texten, die (historischen) Sachanmerkungen bis hin zur Textkritik im engeren Sinne (merke: Eine Reproduktion ohne Kritik ist noch keine Edition!).“

31 Ein nicht unbeträchtlicher Teil von Dargers künstlerischem Hauptwerk, der Story of the Vivian Girls, wurde ediert, es gab etliche Ausstellungen, im Center for Intuitive and Outsider Art in Chicago seit 2008 den Wiederaufbau seines Arbeits- und Wohnzimmers als Rauminstallation, die Einrichtung eines Henry Darger Study Centers am American Folk Art Museum in New York mit entsprechenden Aktivitäten etc. Es stimmt allerdings (ein Beispiel dafür ist auch Adolf Wölfli, dessen umfangreicher Nachlass im Kunstmuseum Bern aus konservatorischen Gründen nicht befriedigend zugänglich gemacht geschweige denn ediert werden kann), dass Art Brut und Outsider Art aufgrund mangelhafter Materialien und Lagerungen oftmals schlechte Erhaltungszustände aufweist, die auch Editionsprobleme nach sich ziehen können.

32 Die von Setz und Lendle angesprochene „unüberschaubare Menge der tatsächlich unmöglichen Literatur, die ihrem Namen Ehre macht und ungeschrieben bleibt", wäre hier mit Blick auf große Formen besonders zu berücksichtigen, zumal das Simulakrum in der (post)modernen Literatur eine wichtige Rolle spielt. So schreibt Borges $1941 \mathrm{im}$ Prolog zu El jardin de senderos que se bifurcan: „Ein mühseliger und strapazierender Unsinn ist es, dicke Bücher zu verfassen; auf fünfhundert Seiten einen Gedanken auszuwalzen, dessen vollkommen ausreichende mündliche Darlegung wenige Minuten beansprucht. Ein besseres Verfahren ist es, so zu tun, als gäbe es diese Bücher bereits, und ein Résumé, einen Kommentar vorzulegen. So machte es Carlyle in Sartor Resartus, so Butler in The Fair Heaven: Werke, behaftet mit der Unvollkommenheit, daß sie eben auch Bücher sind, nicht minder tautologisch als die anderen. Aus größerer Gewitztheit, größerer Unbegabtheit, größerer Faulheit habe ich das Schreiben von Anmerkungen zu imaginären Büchern vorgezogen.“',Desvario laborioso y empobrecedor el de componer vastos libros; el de explayar en quinientas páginas una idea cuya perfecta exposición oral cabe en pocos minutos. Mejor procedimiento es simular que esos libros ya existen y ofrecer un resumen, un comentario. Así procedió Carlyle en Sartor Resartus; asi Butler en The Fair Haven; obras que tienen la imperfección de ser libros también, no menos tautológicos que los otros. Más razonable, más inepto, más haragán, he preferido la escritura de notas sobre libros imaginarios." (BORGEs [1991, Bd. 5, 13]/[1996, Bd. 1, 429]) Vgl. auch aus dem Vorwort zu Prólogos con un prólogo de prólogos von 1975: „Die Durchsicht dieser vergessenen Seiten gab mir den Plan eines anderen - originelleren und besseren - Buchs ein; ich biete ihn denen an, die ihn ausführen möchten. Ich glaube, er verlangt geschicktere Hände und eine Beharrlichkeit, die mich längst verlassen hat. In den 1830ern gab Carlyle in seinem Sartor Resartus vor, ein gewisser deutscher Professor habe einen gelehrten Band über die Philosophie der Kleidung in Druck gegeben, und er übersetzte ihn teilweise und kommentierte ihn, durchaus nicht ohne Einwände. Das Buch, das ich nun im Geiste sehe, ist ähnlicher Art. Es bestünde aus einer Reihe von Vorworten zu Büchern, die es nicht gibt. Es brächte überreichlich exemplarische Zitate aus diesen möglichen Werken. Es gibt Plots, die sich zur beflissenen Ausarbeitung weniger anbieten als zu müßigen Spielen der Phantasie oder zum nachsichtigen Dialog; derlei Plots wären die ungreifbare Substanz dieser Seiten, die nicht geschrieben werden. So schrieben wir vielleicht das Vorwort zu einem Quijote oder Quijano, der nie weiß, ob er ein armes Subjekt ist, das ein von Hexern umzingelter Paladin zu sein träumt, oder ein von Hexern umzingelter Paladin, 
aber wohl letztlich keine, die grundsätzlich unabschließbar oder unmöglich sind. Dass die große Form eine übergroße Lebens- und Lesezeit erfordert, aber auch schon individuelle Schreib- und Produktionszeit so beanspruchen kann, dass davon letztlich sogar Einzelbiographien zerstört werden, auch die von Herausgebern und Editoren, ${ }^{33}$ ist evident, aber nicht unverträglich mit einer Konzeption von Edition, die konserviert, bewahrt, verdauert und tradiert. Die editorische Perspektive ist immer aufs zeitlich große Ganze hin ausgerichtet, auf die longue durée, den long run, so pathetisch es klingt, auf die Ewigkeit.

So kann das die vorliegenden Überlegungen durchziehende antike vita brevis, ars longaDiktum auf Editionen gewendet sogar anders intoniert werden: Vita brevis, sed ars longa. Egal, wie beschränkt und vergeblich alle Einzelbemühungen auch ausfallen wollen: Gerade editorische Praktiken als genuin konservierende und sorgsame, oftmals arbeitsteilig organisierte und unter Einbindung von Institutionen erbrachte, entziehen Texte dem Lauf des Lebens bzw. seiner Kürze und erschließen ihnen eine nahezu überzeitliche Dimension. Denn selbst ungelesene und ungenutzte (große kritische) Editionen sind auf eine Zukunft hin ausgerichtet, in der sie (wieder) von Belang sein und neue Leser finden können. „Was bleibet aber, stiften die Dichter", heißt es bei jemandem, an dessen Werk sich wie bei keinem zweiten editorische Grundsatzfragen entzündeten, ohne die zumindest im deutschsprachigen Raum die Entwicklung der Editorik zu einer Editionswissenschaft und eigenständigen wissenschaftliche Disziplin anders verlaufen wäre. ${ }^{34}$

Die Voraussetzung dafür bleiben aber editorische Operationen - unabdingbar bei großen Formen, die individuelle Speichermöglichkeiten und orale Traditionen immer überschreiten und nur durch externe Speichermedien überhaupt tradiert werden können, sobald sie gewisse Umfänge, Organisationsniveaus und Komplexitäten erreichen, wie sie ohne symbolischen Zeichengebrauch nicht herstellbar sind -, die Dichter liefern sie nicht selbst. Sie können es wohl auch kaum, es ist ein Gemenge aus zusätzlichen Personen und Institutionen, die den Buchmarkt, den Literaturbetrieb, das Buchwesen, das System Literatur bilden. So könnte man überpointiert auch folgern, dass es ohne Editionswissenschaften, ohne Praktiken der Erschließung, Verbesserung, Bewahrung, aber auch Publikation und

der ein armes Subjekt zu sein träumt.“" La revisión de estas páginas olvidadas me ha sugerido el plan de otro libro más original y mejor, que ofrezco a quienes quieran ejecutarlo. Pienso que exige manos más diestras y una tenacidad que ya me ha dejado. Carlyle, hacia mil ochocientos treinta y tantos, simuló en su Sartor Resartus, que cierto profesor alemán habia dado a la imprenta un docto volumen sobre la filosofía de la ropa y lo tradujo parcialmente y lo comentó, no sin algún reparo. El libro que ya estoy entreviendo es de indole análoga. Constaría de una serie de prólogos de libros que no existen. Abundaría en citas ejemplares de esas obras posibles. Hay argumentos que se prestan menos a la escritura laboriosa que a los ocios de la imaginación o al indulgente diálogo, tales argumentos serían la impalpable sustancia de esas páginas que no se escribirán. Prologaríamos, acaso, un Quijote o Quijano que nunca sabe si es un pobre sujeto que sueña ser un paladin cercado de hechiceros o un paladin cercado de hechiceros que sueña ser un probre sujeto. Convendría, por supuesto, eludir la parodia y la sátira, las tramas deberian ser de aquellas que nuestra mente acepta y anhela." (Borges [1991, Bd. 18, 13]/[1996, Bd. 4, 14]).

33 Das Ende der Edition und der Beginn ihres Lebens auf dem Buchmarkt wäre der Tod des Editors; aber keine Edition ist je abgeschlossen. Es gibt, wenn der letzte Band (laut Editionsplan natürlich nur) gedruckt vorliegt, wohl nur zwei Wege: Aufhören - oder weitermachen und wieder von vorne beginnen. Die meisten Editoren machen weiter, d. h. kümmern sich um die Verbesserung von Fehlern. Oder wählen einen anderen Gegenstand.

34 Vgl. Hölderlin (2000) und Hoffmann, Zils (2005). 
Verbreitung literarischer Texte, keine großen Formen und keine Literatur in Übergröße geben kann. ${ }^{35}$ Eine Unedierbarkeit großer Formen ergäbe sich lediglich, wo nicht ediert würde. „Was bleibet aber, stiften die Dichter" - solange sie nur publiziert, herausgegeben und ediert werden.

\section{Literaturverzeichnis}

ALEWYN, Richard (23./24.2.1974, 24): Noch einmal: Hofmannsthal-Ausgabe. In: FAZ.

- (15./16.5.1976): 20000 Blätter von Hofmannsthals Hand. Die kritische Edition seiner Werke hat begonnen. In: SZ.

Bartl, Andrea, Corina Erk, Martin Kraus (Hrsg.) (2019): Verhinderte Meisterwerke. Gescheiterte Projekte in Literatur und Film. Paderborn.

BAYARD, Pierre (2007): Comment parler des livres que l'on n'a pas lus? Paris.

Borges, Jorge Luis (1991): Werke in 20 Bänden. Hrsg. v. G. Haefs, F. Arnold. Frankfurt a. M.

- (1996), Obras completas. Barcelona.

CAHn, Michael (2004, 81-94): Opera Omnia. The Production of Cultural Authority. In: K. Chemla (Hrsg.): History of Science, History of Text. Dordrecht.

Cheshire, Gerard (2019, 30-67): The Language and Writing System of MS408 (Voynich) Explained. In: Romance Studies, 37. Jg., H. 1.

Driesch, Carl, Paul Schlager (1957-1960): Goethe-Bibliographie 1912-1950 (Goedeckes Grundriss IV/5). Berlin, Düsseldorf.

Eco, Umberto (1990): I limiti dell' interpretazione. Mailand.

- (1994): Six Walks in the Fictional Woods. Cambridge, MA. u. a.

Geiger, Ludwig (1912, 231-232): Zur Erinnerung an Bernhard Suphan. In: Goethe-Jahrbuch, 33. Jg.

Goethe, Johann Wolfgang (1790): Faust. Ein Fragment. Ächte Ausgabe. Leipzig. Online-Edition, $<$ http://www.deutschestextarchiv.de/book/show/goethe_faustfragment_1790>, zuletzt: 27.4.2020.

- (1808): Faust. Eine Tragödie. Tübingen. Online-Edition, <http://www.deutschestextarchiv.de/ book/view/goethe_faust01_1808?p=93>, zuletzt: 27.4.2020.

- (1887a): Goethes Faust in ursprünglicher Gestalt nach der Göchhausenschen Handschrift. Hrsg. v. E. Schmidt. Weimar.

- (1887b): Werke. Hrsg. im Auftrage der Großherzogin Sophie von Sachsen. Bd. 14. Faust. Erster Theil. Weimar.

- (1888a): Werke. Hrsg. im Auftrage der Großherzogin Sophie von Sachsen. Bd. 15. Erste Abtheilung. Faust. Zweiter Theil. Weimar.

- (1888b): Werke. Hrsg. im Auftrage der Großherzogin Sophie von Sachsen. Bd. 15. Zweite Abtheilung. Faust. Zweiter Theil.

- (1911): Der Urfaust. Goethes Faust in ursprünglicher Gestalt. Mit Einleitung. Hrsg. v. R. Petsch. Leipzig.

- (2018): Faust Historisch-kritische Edition. Hrsg. v. A. Bohnenkamp, S. Henke, F. Jannidis u. Mitarbeit v. G. Brüning u. a. Frankfurt a. M. u. a., <http://www.faustedition.net>.

Hoffmann, Dierk O., Harald Zils (2005, 199-245): Hölderlin-Editionen. In: Editionen zu deutschsprachigen Autoren als Spiegel der Editionsgeschichte. Hrsg. v. R. Nutt-Kofoth, B. Plachta. Tübingen.

35 Und vielleicht auch umgekehrt; die Geschichte der Philologie ließe sich als Geschichte des Umgangs mit immer größeren und komplexeren Textsammlungen und der editorischen Bewältigung (zu) großer und langer Texte rekonstruieren, bspw. in der antiken Homer-Philologie, die nicht nur Fragen der Authentizität von Teilen der Ilias und Odyssee erstmals diskutierte, sondern deren Aufteilung in Gesänge vornahm, textkritische Operationen normierte und auch graphisch konventionalisierte, vgl. PFEIFFER (1968). 
Hofmannsthal, Hugo von (1975-202X): Sämtliche Werke. Kritische Ausgabe. Veranstaltet vom Freien Deutschen Hochstift. Hrsg. v. A. Bohnenkamp (ab 2004), H. O. Burger (1975-1977) u. a. Frankfurt a. M.

HÖLDERLin, Friedrich (2000, 805): Andenken. In: Sämtliche Werke. Frankfurter Ausgabe. Hrsg. v. D. E. Sattler. Bd. 8. Frankfurt a. M. 2000.

Hunger, Herbert u. a. (Hrsg.) (1961): Geschichte der Textüberlieferung der antiken und mittelalterlichen Literatur. Bd. 1. Antikes und mittelalterliches Buch- und Schriftenwesen. Überlieferungsgeschichte der antiken Literatur. Mit einem Vorwort von M. Bodmer. Redaktion M. Meier, F. Hindermann, A. Schindler. Zürich.

JoYCE, James (1939): Finnegans Wake. London.

Kamzelak, Roland, Rüdiger NutT-Kofoth, Bodo Plachta (Hrsg.) (2011): Neugermanistische Editoren im Wissenschaftskontext. Berlin, Boston.

Langosch, Karl u. a. (Hrsg.) (1964): Geschichte der Textüberlieferung der antiken und mittelalterlichen Literatur. Bd. 2. Überlieferungsgeschichte der mittelalterlichen Literatur. Redaktion G. Ineichen, A. Schindler, D. Bodmer. Zürich.

- (1964, 9-185): Überlieferungsgeschichte der mittellateinischen Literatur. In: LANGOSCH u. a. (1964).

Lendle, Jo, Clemens Setz (2015, 1-3): Vorwort. In: Akzente. Zeitschrift für Literatur, 62. Jg., H. 1.

Metz, Bernhard (2008, 249-269): Bibliomania and the Folly of Reading. In: Comparative Critical Studies 5, H. 2-3.

- (i. Dr.): Bookishness and the Body of the Book/the Body of the Reader. On the Usages of Books. In: V. Hildebrand-Schat (Hrsg.): The Book as an Enhanced Space of Conception and Communication. Leiden.

Mittelmeier, Martin (Hrsg.) (2006): Ungeschriebene Werke. Wozu Goethe, Flaubert, Jandl und all die anderen nicht gekommen sind. München.

MoretTi, Franco (Januar/Februar 2000, 54-68): Conjectures on World Literature. In: New Left Review 1.

MusiL, Robert (1930): Der Mann ohne Eigenschaften. Berlin. Online-Edition, <http://musilonline.at./ musiltext/der-mann-ohne-eigenschaften-2/moe2-teil-2-100-109>, zuletzt: 27.4.2020.

- (2009, Transkriptionen/Mappe VIII/5/43): Klagenfurter Ausgabe. Kommentierte Edition sämtlicher Werke, Briefe und nachgelassener Schriften. Mit Transkriptionen und Faksimiles aller Handschriften. Hrsg. v. W. Fanta, K. Amann, K. Corino. Klagenfurt, Wien.

Pehnt, Annette, Friedemann Holder, Michael Staiger (Hrsg.) (2014): Die Bibliothek der ungeschriebenen Bücher. München, Zürich.

Pfeiffer, Rudolf (1968): History of Classical Scholarship. From the Beginnings to the End of the Hellenistic Age. Oxford.

Polgar, Alfred (1984, 369-373): Die kleine Form. In: Ders.: Kleine Schriften, Bd. 3. Irrlicht. Reinbek b. H.

SAHLE, Patrick (2007, 234-249): Digitale Edition. In: F. Jannidis, H. Kohle, M. Rehbein (Hrsg.): Digital Humanities. Eine Einführung. Mit Abbildungen und Grafiken. Stuttgart.

Schmidt, Arno (1988, 30-31): Ich bin erst Sechzig. (Niederschrift 25. Juni 1955). In: Bargfelder Ausgabe. Werkgruppe I. Romane, Erzählungen, Gedichte, Juvenilia. Bd. 4. Kleinere Erzählungen, Gedichte, Juvenilia. Hrsg. v. d. Arno Schmidt Stiftung. Zürich.

- (1995, 87-92): Julianische Tage. (Niederschrift 15./16. Juni 1961). In: Bargfelder Ausgabe. Werkgruppe III. Essays und Biographisches. Bd. 4. Essays und Aufsätze II. Hrsg. v. d. Arno Schmidt Stiftung. Zürich.

Serafini, Luigi (1981): Codex Seraphinianus. Aloisius Seraphini [d. i. Luigi Serafini] invenit et pinxit. Mailand.

Shillingsburg, Peter L. (2017a, vii-xii): Preface and Acknowledgments. In: Ders.: Textuality and Knowledge. Essays. University Park, PA. 
- (2017b, 115-133): How Literary Works Exist. In: Ders.: Textuality and Knowledge. Essays. University Park, PA.

- (2017c, 134-144): Convenient Scholarly Editions. In: Ders.: Textuality and Knowledge. Essays. University Park, PA.

WeInRICH, Harald (1984, 80-99): Lesen - schneller lesen - langsamer lesen. In: Neue Rundschau, 95. Jg., H. 3.

\section{Abstract}

Große Formen und übergroße Literatur sind nicht nur an Schriftlichkeit und symbolischen Zeichengebrauch gekoppelt, sie bedürfen auch stärker als kurze eines personellen und institutionellen Umfelds (Literaturbetrieb, Buchwesen, Buchmarkt), um erstellt, publiziert und rezipiert werden zu können. Ihre Textüberlieferung ist ohne editorische Transmission undenkbar. Doch gibt es auch eine Unedierbarkeit großer Formen, und tendieren diese zu unabschließbaren oder unmöglichen Editionsunternehmungen?

Large literary genres and lengthy literature not only depend on the use of literacy and symbolic signs, they also require a huge personal and institutional environment (book industry, book market) in order to be created, published, and received. Their textual transmission is unthinkable without editorial interventions. But is there also an uneditability of literary large genres, and do they tend to be unfinishable or to provoke impossible edition projects?

Keywords: Edierbarkeit, Edition, Große Formen, Lesbarkeit, Nutzbarkeit

Anschrift des Verfassers: Dr. Bernhard Metz, Universität Bern, Institut für Medizingeschichte, Bühlstrasse 26, 3012 Bern, Postfach CH-3000 Bern 9, <bernhard. metz@img.unibe.ch> 